\title{
Ray tracing and geodesic deviation of the SH and SV reference rays in a heterogeneous generally anisotropic medium which is approximately uniaxial
}

\author{
LudĚK KLIMEŠ ANd Petr Bulant
}

Department of Geophysics, Faculty of Mathematics and Physics, Charles University, Ke Karlovu 3, 12116 Praha 2, Czech Republic (http://sw3d.cz/staff/klimes.htm, http://sw3d.cz/staff/bulant.htm)

Received: October 1, 2015; Revised: October 12, 2016; Accepted: October 18, 2016

\begin{abstract}
The coupling ray theory is usually applied to anisotropic common reference rays, but it is more accurate if it is applied to reference rays which are closer to the actual wave paths. If we know that a medium is close to uniaxial (transversely isotropic), it may be advantageous to trace reference rays which resemble the SHwave and $S V$-wave rays. This paper is devoted to defining and tracing these $S H$ and $S V$ reference rays of elastic $S$ waves in a heterogeneous generally anisotropic medium which is approximately uniaxial (approximately transversely isotropic), and to the corresponding equations of geodesic deviation (dynamic ray tracing). All presented equations are simultaneously applicable to ordinary and extraordinary reference rays of electromagnetic waves in a bianisotropic medium with the symmetric constitutive matrix which is approximately uniaxially anisotropic. The improvement of the coupling-ray-theory seismograms calculated along the proposed $S H$ and $S V$ reference rays, compared to the coupling-ray-theory seismograms calculated along the anisotropic common reference rays, has already been numerically demonstrated by the authors in four approximately uniaxial velocity models.
\end{abstract}

Keywords: wave propagation, elastic anisotropy, electromagnetic bianisotropy, heterogeneous media, wave coupling, approximately uniaxial (approximately transversely isotropic) anisotropic medium, reference symmetry axis, SH (ordinary) and SV (extraordinary) reference rays 


\section{INTRODUCTION}

\subsection{Elastic waves}

Consider a line intersection singularity at the slowness surface in an uniaxial (transversely isotropic) elastic medium. In this special case, the $\mathrm{SH}-$ wave and $\mathrm{SV}-$ wave rays cross smoothly this intersection singularity. However, this intersection singularity is unstable, and any perturbation from transverse isotropy causes this intersection singularity to split (Crampin, 1981). The slower S-wave slowness sheet then separates from the faster $\mathrm{S}$-wave slowness sheet, forming smooth but very sharp edges on both sheets (Bulant and Kliměs, 2014; Klimeš and Bulant, 2014b).

When the slowness vector of a ray passes smoothly through a split intersection singularity, the ray-velocity vector rapidly changes its direction and creates a sharp bend in the ray. This sharp bend is connected with a rapid rotation of the eigenvectors of the Christoffel matrix. The sharply bent rays thus cannot describe the correct wave propagation.

The actual $\mathrm{S}$ waves do not propagate along the sharply bent rays, but tunnel smoothly through a split intersection singularity (Klimeš and Bulant, 2012, 2016). If a medium is close to uniaxial (transversely isotropic), the actual S-wave paths resemble the $\mathrm{SH}$-wave and $\mathrm{SV}$-wave rays.

On the one hand, the coupling ray theory (Chapman and Shearer, 1989; Coates and Chapman, 1990; Bulant and Kliměs, 2002) is usually applied to anisotropic common reference rays (Bakker, 2002; Klimeš and Bulant, 2004, 2006; Kliměs, 2006; Bulant and Klimeš, 2008). On the other hand, the coupling ray theory is more accurate if it is applied to reference rays which are closer to the actual S-wave paths (Klimeš and Bulant, 2014a, 2015; Bulant and Klimeř, 2017).

If we know that a medium is close to uniaxial, it is reasonable to trace the reference rays which tunnel smoothly through a split intersection singularity and resemble the $\mathrm{SH}$-wave and $\mathrm{SV}$-wave rays. This paper is devoted to defining and tracing these $\mathrm{SH}$ and $\mathrm{SV}$ reference rays in a heterogeneous generally anisotropic medium which is approximately uniaxial, and to the corresponding equations of geodesic deviation (dynamic ray tracing). The proposed equations are especially useful in approximately uniaxial media, but can also be applied to media which are not close to uniaxial.

Whether an elastic medium is close to uniaxial can be determined by the method of Klimeš (2015, 2016a). This method also determines the direction of the corresponding reference symmetry axis in terms of the reference symmetry vector.

The improvement of the coupling-ray-theory seismograms calculated along the proposed SH and SV reference rays, compared to the coupling-ray-theory seismograms calculated along the anisotropic common reference rays, has already been numerically demonstrated in four approximately uniaxial velocity models by Klime š and Bulant (2014a, 2015) and Bulant and Klimeš (2017).

On the one hand, the proposed SH and SV reference rays are extremely important as the reference rays for the coupling ray theory in an approximately uniaxial elastic medium with a split intersection singularity at the slowness surface (Klimers and 
Bulant, 2015; Bulant and Klimeš, 2017). On the other hand, if there is no split intersection singularity at the slowness surface, we can use the anisotropic-raytheory rays successfully as the reference rays for the coupling ray theory instead of the SH and SV reference rays (Klimeš and Bulant, 2014a).

\subsection{Electromagnetic waves}

In the propagation of electromagnetic waves, a rotationally invariant anisotropic medium (Kliměs, 2017a) which is transversely isotropic is referred to as uniaxial rather than transversely isotropic. The rays in an uniaxial anisotropic electromagnetic medium are referred to as ordinary rather than SH, and extraordinary rather than SV.

The coupling ray theory for electromagnetic waves in its quasi-isotropic approximation was proposed by Kravtsov (1968). In order to increase the accuracy of the coupling ray theory, Naida (1977, 1979) and Fuki et al. (1998, Sec. 3.2) proposed to apply the coupling ray theory for electromagnetic waves to ordinary and extraordinary reference rays.

All equations presented in this paper are applicable to electromagnetic waves in bianisotropic media with symmetric constitutive matrices which are approximately uniaxially anisotropic. Using the Weyl gauge (zero electric potential), we just need to replace the elastic Christoffel matrix by the analogous Kelvin-Christoffel matrix corresponding to the Maxwell equations for the magnetic vector potential in electromagnetic bianisotropic media, see Klimeš (2010, 2016b,c). We then read generally anisotropic as bianisotropic, approximately uniaxial as approximately uniaxially anisotropic, $S H$ as ordinary and $S V$ as extraordinary. The two S-wave eigenvectors and the corresponding eigenvalues of the Christoffel matrix then stand for the two eigenvectors and two eigenvalues of the Kelvin-Christoffel matrix corresponding to electromagnetic waves, while the $\mathrm{P}$-wave eigenvector and eigenvalue of the Christoffel matrix stand for the third eigenvector and the corresponding eigenvalue of the Kelvin-Christoffel matrix which do not correspond to a propagating electromagnetic wave.

Whether a bianisotropic or anisotropic electromagnetic medium is close to rotationally invariant or uniaxial can be determined by the method of Klimeš (2017b). This method also determines the direction of the corresponding reference symmetry axis in terms of the reference symmetry vector.

However, since there is no line intersection singularity at the slowness surface in an uniaxial anisotropic electromagnetic medium, we can probably successfully use the anisotropic-ray-theory rays as the reference rays for the coupling ray theory in a bianisotropic medium which is approximately uniaxially anisotropic instead of the proposed ordinary and extraordinary reference rays.

\subsection{Notation}

The lower-case Roman indices take values 1, 2 and 3. The upper-case Roman indices take values 1 and 2 . Indices in parentheses are used to index the eigenvalues and eigenvectors of the Christoffel matrix. The Einstein summation over repetitive indices is used throughout the paper. 


\section{Klimeš and P. Bulant}

\section{HAMILTONIAN FUNCTION AND HAMILTON'S EQUATIONS OF RAYS}

We consider a smooth manifold (differentiable manifold), and coordinates $x^{i}$ of its coordinate chart. At each point $x^{i}$, the cotangent space contains covariant vectors $p_{i}$ such as the gradients of functions. We consider Hamiltonian function $H\left(x^{i}, p_{j}\right)$, which is a real-valued function of coordinates $x^{i}$ and of covariant vector $p_{j}$ from the cotangent space at point $x^{i}$, and which is differentiable within its definition domain. The Hamiltonian function may be represented by any reasonably smooth function of $x^{i}$ and $p_{j}$.

Hamilton's equations (equations of geodesics, equations of rays, ray tracing equations) read

$$
\begin{gathered}
\frac{\mathrm{d}}{\mathrm{d} \gamma} x^{i}=\frac{\partial H}{\partial p_{i}}\left(x^{m}, p_{n}\right), \\
\frac{\mathrm{d}}{\mathrm{d} \gamma} p_{i}=-\frac{\partial H}{\partial x^{i}}\left(x^{m}, p_{n}\right) .
\end{gathered}
$$

Hamilton (1837) referred to these equations as the general equations of rays. The meaning of the independent parameter $\gamma$ along the ray and the sensitivity of the ray to the initial conditions depend on the form of the Hamiltonian function (Klime 2013).

We define vectors

$$
X_{\alpha}^{i}=\frac{\partial x^{i}}{\partial \gamma^{\alpha}}
$$

and

$$
P_{i \alpha}=\frac{\partial p_{i}}{\partial \gamma^{\alpha}}
$$

representing the geodesic deviation corresponding to some parameter $\gamma^{\alpha}$ parametrizing the initial conditions for the rays (geodesics). Since derivatives $\frac{\mathrm{d}}{\mathrm{d} \gamma}$ and $\frac{\partial}{\partial \gamma^{\alpha}}$ commute, the equations for $X_{\alpha}^{i}$ and $P_{i \alpha}$ are obtained by differentiating Hamilton's equations (1)-(2) with respect to $\gamma^{\alpha}$. The resulting Hamiltonian equations of geodesic deviation (paraxial ray equations, dynamic ray tracing equations) derived by Červený (1972) read

$$
\begin{aligned}
\frac{\mathrm{d}}{\mathrm{d} \gamma} X_{\alpha}^{i} & =\frac{\partial^{2} H}{\partial p_{i} \partial x^{j}}\left(x^{m}, p_{n}\right) X_{\alpha}{ }_{\alpha}+\frac{\partial^{2} H}{\partial p_{i} \partial p_{j}}\left(x^{m}, p_{n}\right) P_{j \alpha}, \\
\frac{\mathrm{d}}{\mathrm{d} \gamma} P_{i \alpha} & =-\frac{\partial^{2} H}{\partial x^{i} \partial x^{j}}\left(x^{m}, p_{n}\right) X_{\alpha}^{j}-\frac{\partial^{2} H}{\partial p_{j} \partial x^{i}}\left(x^{m}, p_{n}\right) P_{j \alpha} .
\end{aligned}
$$

Note that Hamiltonian equations (5)-(6) of geodesic deviation may differ for different Hamiltonian functions corresponding to equal rays.

In the following sections, we propose reference Hamiltonian function $H\left(x^{i}, p_{j}\right)=$ $H_{\mathrm{SH}}\left(x^{i}, p_{j}\right)$ for tracing the $\mathrm{SH}$ or ordinary reference rays, and reference Hamiltonian function $H\left(x^{i}, p_{j}\right)=H_{\mathrm{SV}}\left(x^{i}, p_{j}\right)$ for tracing the $\mathrm{SV}$ or extraordinary reference rays in order to increase the accuracy of the coupling ray theory. 


\section{REFERENCE SH AND SV HAMILTONIAN FUNCTIONS}

We assume that a given heterogeneous bianisotropic or anisotropic medium with the symmetric constitutive or stiffness matrix is approximately uniaxially anisotropic with respect to given reference symmetry vector $t_{i}$. For the sake of simplicity, we consider the reference symmetry vector constant in this paper. However, the presented equations can simply be generalized to a generally heterogeneous dependence of the reference symmetry vector on the spatial coordinates.

For the sake of simplicity, we assume the Christoffel matrix $\Gamma_{j k}$ symmetric in this paper.

We project the reference symmetry vector onto the plane defined by two S-wave eigenvectors $g_{i(1)}, g_{i(2)}$ of the Christoffel matrix $\Gamma_{j k}$, and introduce the reference SV polarization in the direction of this projection. The unit reference SV polarization vector then reads

$$
g_{i \mathrm{SV}}=\frac{g_{i(A)} g_{k(A)} t_{k}}{\sqrt{t_{r} g_{r(N)} g_{s(N)} t_{s}}} .
$$

We cannot use this definition in a special singular case when the denominator of definition (7) is zero within the rounding errors at a point of the traced ray. In this case, we can replace reference symmetry vector $t_{k}$ in definition (7) and all other presented equations by the reference SV polarization vector determined at the previous point of the traced ray. Note that we do not need to introduce the reference $\mathrm{SH}$ polarization vector whose definition is slightly more cumbersome and thus less suitable for differentiation.

We define twice the reference $S V$ Hamiltonian function $G_{\mathrm{SV}}=2 H_{\mathrm{SV}}\left(x^{m}, p_{n}\right)$ as

$$
G_{\mathrm{SV}}=g_{j \mathrm{SV}} \Gamma_{j k} g_{k \mathrm{SV}} .
$$

We then define twice the reference $S H$ Hamiltonian function $G_{\mathrm{SH}}=2 H_{\mathrm{SH}}\left(x^{m}, p_{n}\right)$ in terms of twice the reference SV Hamiltonian function as

$$
G_{\mathrm{SH}}=G_{(1)}+G_{(2)}-G_{\mathrm{SV}}
$$

where $G_{(1)}$ and $G_{(2)}$ are the S-wave eigenvalues of the Christoffel matrix.

As we need to handle both the derivatives with respect to spatial coordinates $x^{m}$ and slowness-vector components $p_{n}$, we denote any partial phase-space derivative $\partial / \partial x^{m}$ or $\partial / \partial p_{n}$ by ${ }^{\prime}$ or * .

The phase-space derivatives of twice the reference SH Hamiltonian function can then be expressed as

$$
G_{\mathrm{SH}}^{\prime}=G_{(1)}^{\prime}+G_{(2)}^{\prime}-G_{\mathrm{SV}}^{\prime}
$$

and

$$
G_{\mathrm{SH}}^{\prime \star}=G_{(1)}^{\prime \star}+G_{(2)}^{\prime \star}-G_{\mathrm{SV}}^{\prime \star}
$$




\section{Klimeš and P. Bulant}

For the phase-space derivatives of the S-wave eigenvalues of the Christoffel matrix refer to Klimeš (2006, Eqs 22, 23). Hereinafter, we shall concentrate on the reference SV Hamiltonian function and its first-order and second-order phase-space derivatives.

We insert projection (7) into definition (8) and obtain

$$
G_{\mathrm{SV}}=\frac{t_{i} g_{i(K)} g_{j(K)} \Gamma_{j k} g_{k(L)} g_{l(L)} t_{l}}{t_{r} g_{r(M)} g_{s(M)} t_{s}} .
$$

Since the eigenvectors of the Christoffel matrix are orthonormal,

$$
g_{i(a)} g_{j(a)}=\delta_{i j},
$$

we insert identity

$$
g_{i(K)} g_{j(K)}=\delta_{i j}-g_{i(3)} g_{j(3)}
$$

into twice the reference SV Hamiltonian function (12), and obtain expression

$$
G_{\mathrm{SV}}=\frac{t_{i}\left(\delta_{i j}-g_{i(3)} g_{j(3)}\right) \Gamma_{j k}\left(\delta_{k l}-g_{k(3)} g_{l(3)}\right) t_{l}}{t_{r}\left(\delta_{r s}-g_{r(3)} g_{s(3)}\right) t_{s}} .
$$

After multiplication, this expression becomes

$$
G_{\mathrm{SV}}=\frac{t_{i} \Gamma_{i j} t_{j}-\left(t_{i} g_{i(3)}\right)^{2} G_{(3)}}{t_{s} t_{s}-\left(t_{s} g_{s(3)}\right)^{2}}
$$

where

$$
G_{(3)}=g_{i(3)} \Gamma_{i j} g_{j(3)}
$$

is the $\mathrm{P}$-wave eigenvalue of the Christoffel matrix. For simpler differentiation, we express twice the reference SV Hamiltonian function (16) as

$$
G_{\mathrm{SV}}=\frac{t_{i} \Gamma_{i j} t_{j}-G_{(3)} t_{r} t_{r}}{t_{s} t_{s}-\left(t_{s} g_{s(3)}\right)^{2}}+G_{(3)} .
$$

\section{PHASE-SPACE DERIVATIVES}

\section{OF THE REFERENCE SV HAMILTONIAN FUNCTION}

\subsection{Differentiating}

the reference SV Hamiltonian function

We now differentiate relation (18),

$$
G_{\mathrm{SV}}^{\prime}=\frac{t_{i} \Gamma_{i j}^{\prime} t_{j}-G_{(3)}^{\prime} t_{r} t_{r}}{t_{s} t_{s}-\left(t_{s} g_{s(3)}\right)^{2}}+G_{(3)}^{\prime}+2 \frac{t_{i} \Gamma_{i j} t_{j}-G_{(3)} t_{r} t_{r}}{\left[t_{s} t_{s}-\left(t_{s} g_{s(3)}\right)^{2}\right]^{2}} t_{m} g_{m(3)} t_{n} g_{n(3)}^{\prime}
$$

We again differentiate relation (19),

$$
\begin{aligned}
G_{\mathrm{SV}}^{\prime \star} & =\frac{t_{i} \Gamma_{i j}^{\prime \star} t_{j}-G_{(3)}^{\prime \star} t_{r} t_{r}}{t_{s} t_{s}-\left(t_{s} g_{s(3)}\right)^{2}}+G_{(3)}^{\prime \star}+2 \frac{t_{i} \Gamma_{i j}^{\prime} t_{j}-G_{(3)}^{\prime} t_{r} t_{r}}{\left[t_{s} t_{s}-\left(t_{s} g_{s(3)}\right)^{2}\right]^{2}} t_{m} g_{m(3)} t_{n} g_{n(3)}^{\star} \\
& +2 \frac{t_{i} \Gamma_{i j}^{\star} t_{j}-G_{(3)}^{\star} t_{r} t_{r}}{\left[t_{s} t_{s}-\left(t_{s} g_{s(3)}\right)^{2}\right]^{2}} t_{m} g_{m(3)} t_{n} g_{n(3)}^{\prime}+8 \frac{t_{i} \Gamma_{i j} t_{j}-G_{(3)} t_{r} t_{r}}{\left[t_{s} t_{s}-\left(t_{s} g_{s(3)}\right)^{2}\right]^{3}}\left(t_{r} g_{r(3)}\right)^{2} t_{m} g_{m(3)}^{\prime} t_{n} g_{n(3)}^{\star} \\
& +2 \frac{t_{i} \Gamma_{i j} t_{j}-G_{(3)} t_{r} t_{r}}{\left[t_{s} t_{s}-\left(t_{s} g_{s(3)}\right)^{2}\right]^{2}}\left(t_{m} g_{m(3)}^{\prime} t_{n} g_{n(3)}^{\star}+t_{m} g_{m(3)} t_{n} g_{n(3)}^{\prime \star}\right) .
\end{aligned}
$$


We insert relation (18) and identity

$$
t_{s} t_{s}-\left(t_{s} g_{s(3)}\right)^{2}=t_{r} g_{r(M)} g_{s(M)} t_{s}
$$

into relation (19),

$$
G_{\mathrm{SV}}^{\prime}=\frac{t_{i} \Gamma_{i j}^{\prime} t_{j}-G_{(3)}^{\prime} t_{r} t_{r}}{t_{r} g_{r(M)} g_{s(M)} t_{s}}+G_{(3)}^{\prime}-2 \frac{G_{(3)}-G_{\mathrm{SV}}}{t_{r} g_{r(M)} g_{s(M)} t_{s}} t_{m} g_{m(3)} t_{n} g_{n(3)}^{\prime},
$$

and into relation $(20)$,

$$
\begin{aligned}
G_{\mathrm{SV}}^{\prime \star} & =\frac{t_{i} \Gamma_{i j}^{\prime \star} t_{j}-G_{(3)}^{\prime \star} t_{r} t_{r}}{t_{r} g_{r(M)} g_{s(M)} t_{s}}+G_{(3)}^{\prime \star}+2 \frac{t_{i} \Gamma_{i j}^{\prime} t_{j}-G_{(3)}^{\prime} t_{r} t_{r}}{\left(t_{r} g_{r(M)} g_{s(M)} t_{s}\right)^{2}} t_{m} g_{m(3)} t_{n} g_{n(3)}^{\star} \\
& +2 \frac{t_{i} \Gamma_{i j}^{\star} t_{j}-G_{(3)}^{\star} t_{r} t_{r}}{\left(t_{r} g_{r(M)} g_{s(M)} t_{s}\right)^{2}} t_{m} g_{m(3)} t_{n} g_{n(3)}^{\prime}-8 \frac{G_{(3)}-G_{\mathrm{SV}}}{\left(t_{r} g_{r(M)} g_{s(M)} t_{s}\right)^{2}}\left(t_{r} g_{r(3)}\right)^{2} t_{m} g_{m(3)}^{\prime} t_{n} g_{n(3)}^{\star} \\
& -2 \frac{G_{(3)}-G_{\mathrm{SV}}}{t_{r} g_{r(M)} g_{s(M)} t_{s}}\left(t_{m} g_{m(3)}^{\prime} t_{n} g_{n(3)}^{\star}+t_{m} g_{m(3)} t_{n} g_{n(3)}^{\prime \star}\right)
\end{aligned}
$$

\subsection{Transformation into the eigenvectors of the Christoffel matrix}

We define the elements of the Christoffel matrix and of its phase--space derivatives with respect to the eigenvectors of the Christoffel matrix as

$$
\begin{gathered}
\Gamma_{(a b)}=g_{i(a)} \Gamma_{i j} g_{j(b)}, \\
\Gamma_{(a b)}^{\prime}=g_{i(a)} \Gamma_{i j}^{\prime} g_{j(b)}
\end{gathered}
$$

and

$$
\Gamma_{(a b)}^{\prime \star}=g_{i(a)} \Gamma_{i j}^{\prime \star} g_{j(b)}
$$

Note that $\Gamma_{(a b)}$ is a diagonal matrix with diagonal elements

$$
\Gamma_{(a a)}=G_{(a)},
$$

and that the first-order phase-space derivatives of the eigenvalues of the Christoffel matrix represent the diagonal elements of symmetric matrix $\Gamma_{(a b)}^{\prime}($ Klime ̌s, 2006, Eq. 22),

$$
G_{(a)}^{\prime}=\Gamma_{(a a)}^{\prime}
$$

We define the components

$$
t_{(a)}=t_{n} g_{n(a)}
$$

of reference symmetry vector $t_{i}$ with respect to the eigenvectors of the Christoffel matrix.

We insert definitions (24) and (29) into definition (12) and obtain relation

$$
G_{\mathrm{SV}}=\frac{t_{(A)} \Gamma_{(A B)} t_{(B)}}{t_{(S)} t_{(S)}}
$$




\section{Klimeš and P. Bulant}

which can be expressed considering Eq. (27) as

$$
G_{\mathrm{SV}}=\frac{\sum_{A} G_{(A)}\left(t_{(A)}\right)^{2}}{t_{(S)} t_{(S)}} .
$$

We insert definitions (25) and (29) and relation (28) into relation (22), and obtain

$$
G_{\mathrm{SV}}^{\prime}=\frac{t_{(a)} \Gamma_{(a b)}^{\prime} t_{(b)}-\left(t_{(3)}\right)^{2} \Gamma_{(33)}^{\prime}}{t_{(S)} t_{(S)}}-2 \frac{G_{(3)}-G_{\mathrm{SV}}}{t_{(S)} t_{(S)}} t_{(3)} t_{n} g_{n(3)}^{\prime}
$$

We insert definitions (25), (26) and (29) and relation (28) into relation (23), and arrive at

$$
\begin{aligned}
G_{\mathrm{SV}}^{\prime \star} & =\frac{t_{(a)} \Gamma_{(a b)}^{\prime \star} t_{(b)}-\left(t_{(3)}\right)^{2} \Gamma_{(33)}^{\prime \star}}{t_{(S)} t_{(S)}}+2 \frac{t_{(a)} \Gamma_{(a b)}^{\prime} t_{(b)}-\Gamma_{(33)}^{\prime} t_{(r)} t_{(r)}}{\left(t_{(S)} t_{(S)}\right)^{2}} t_{(3)} t_{n} g_{n(3)}^{\star} \\
& +2 \frac{t_{(a)} \Gamma_{(a b)}^{\star} t_{(b)}-\Gamma_{(33)}^{\star} t_{(r)} t_{(r)}}{\left(t_{(S)} t_{(S)}\right)^{2}} t_{(3)} t_{n} g_{n(3)}^{\prime}-8 \frac{G_{(3)}-G_{\mathrm{SV}}}{\left(t_{(S)} t_{(S)}\right)^{2}}\left(t_{(3)}\right)^{2} t_{m} g_{m(3)}^{\prime} t_{n} g_{n(3)}^{\star} \\
& -2 \frac{G_{(3)}-G_{\mathrm{SV}}}{t_{(S)} t_{(S)}}\left(t_{m} g_{m(3)}^{\prime} t_{n} g_{n(3)}^{\star}+t_{(3)} t_{n} g_{n(3)}^{\prime \star}\right),
\end{aligned}
$$

which can be expressed as

$$
\begin{aligned}
G_{\mathrm{SV}}^{\prime \star} & =\frac{t_{(a)} \Gamma_{(a b)}^{\prime \star} t_{(b)}-\left(t_{(3)}\right)^{2} G_{(3)}^{\prime \star}}{t_{(S)} t_{(S)}}+2 \frac{t_{(a)} \Gamma_{(a b)}^{\prime} t_{(b)}-\Gamma_{(33)}^{\prime} t_{(r)} t_{(r)}}{\left(t_{(S)} t_{(S)}\right)^{2}} t_{(3)} t_{n} g_{n(3)}^{\star} \\
& +2 \frac{t_{(a)} \Gamma_{(a b)}^{\star} t_{(b)}-\Gamma_{(33)}^{\star} t_{(r)} t_{(r)}}{\left(t_{(S)} t_{(S)}\right)^{2}} t_{(3)} t_{n} g_{n(3)}^{\prime}-2 \frac{G_{(3)}-G_{\mathrm{SV}}}{t_{(S)} t_{(S)}}\left(1+4 \frac{\left(t_{(3)}\right)^{2}}{t_{(S)} t_{(S)}}\right) t_{m} g_{m(3)}^{\prime} t_{n} g_{n(3)}^{\star} \\
& -2 \frac{G_{(3)}-G_{\mathrm{SV}}}{t_{(S)} t_{(S)}} t_{(3)} t_{n} g_{n(3)}^{\prime \star} .
\end{aligned}
$$

We now have to express the second-order phase-space derivatives of the eigenvalues of the Christoffel matrix and the first-order and second-order phase-space derivatives of the eigenvectors of the Christoffel matrix in relations (32) and (34) in terms of the phase-space derivatives of the Christoffel matrix.

\subsection{Second-order phase-space derivatives of the eigenvalues of the Christoffel matrix}

The expression for the second-order phase-space derivatives of the eigenvalues of the Christoffel matrix is (Klimers, 2006, Eq. 23)

$$
G_{(a)}^{\prime \star}=\Gamma_{(a a)}^{\star \star}+2 \sum_{b \neq a} \frac{\Gamma_{(a b)}^{\prime} \Gamma_{(a b)}^{\star}}{G_{(a)}-G_{(b)}}
$$

which for $a=3$ reads

$$
G_{(3)}^{\prime \star}=\Gamma_{(33)}^{\prime \star}+2 \sum_{B} \frac{\Gamma_{(B 3)}^{\prime} \Gamma_{(B 3)}^{\star}}{G_{(3)}-G_{(B)}} .
$$

This expression can be inserted into relation (34). 
$S H$ and $S V$ reference rays in an approximately uniaxial generally anisotropic medium

4.4. First-order phase-space derivatives

of the eigenvectors of the Christoffel matrix

The first-order phase-space derivatives of the eigenvectors of the Christoffel matrix can be expressed as (Kliměs, 2006, Eq. 17)

$$
g_{i(a)}^{\prime}=\sum_{b \neq a} g_{i(b)} \frac{g_{j(b)} \Gamma_{j k}^{\prime} g_{k(a)}}{G_{(a)}-G_{(b)}},
$$

which for $a=3$ with definition $(25)$ reads

$$
g_{i(3)}^{\prime}=\sum_{B} g_{i(B)} \frac{\Gamma_{(B 3)}^{\prime}}{G_{(3)}-G_{(B)}} .
$$

We multiply relation (38) by reference symmetry vector $t_{i}$ and obtain expression

$$
t_{n} g_{n(3)}^{\prime}=\sum_{A} t_{(A)} \frac{\Gamma_{(A 3)}^{\prime}}{G_{(3)}-G_{(A)}}
$$

which can be inserted into relations (32) and (34).

4.5. Second-order phase-space derivatives

of the eigenvectors of the Christoffel matrix

The second-order phase-space derivatives of the eigenvectors of the Christoffel matrix can be obtained by twice differentiating the Christoffel equation (Klimeř, 2006, Eq. 11),

$$
g_{i(a)}^{\prime \star} G_{(a)}+g_{i(a)}^{\prime} G_{(a)}^{\star}+g_{i(a)}^{\star} G_{(a)}^{\prime}+g_{i(a)} G_{(a)}^{\prime \star}=\Gamma_{i j}^{\prime \star} g_{j(a)}+\Gamma_{i j}^{\prime} g_{j(a)}^{\star}+\Gamma_{i j}^{\star} g_{j(a)}^{\prime}+\Gamma_{i j} g_{j(a)}^{\prime \star} .
$$

We multiply relation (40) for $a=3$ by eigenvector $g_{i(C)}$ of the Christoffel matrix,

$$
\begin{aligned}
g_{i(C)} g_{i(3)}^{\prime \star} G_{(3)} & +g_{i(C)} g_{i(3)}^{\prime} G_{(3)}^{\star}+g_{i(C)} g_{i(3)}^{\star} G_{(3)}^{\prime} \\
& =g_{i(C)} \Gamma_{i j}^{\prime \star} g_{j(3)}+g_{i(C)} \Gamma_{i j}^{\prime} g_{j(3)}^{\star}+g_{i(C)} \Gamma_{i j}^{\star} g_{j(3)}^{\prime}+g_{i(C)} \Gamma_{i j} g_{j(3)}^{\prime \star} .
\end{aligned}
$$

We then express two components of the second-order phase-space derivatives of the eigenvector of the Christoffel matrix as

$$
g_{i(C)} g_{i(3)}^{\prime \star}=\frac{g_{i(C)} \Gamma_{i j}^{\prime \star} g_{j(3)}+g_{i(C)} \Gamma_{i j}^{\prime} g_{j(3)}^{\star}+g_{i(C)} \Gamma_{i j}^{\star} g_{j(3)}^{\prime}-g_{i(C)} g_{i(3)}^{\prime} G_{(3)}^{\star}-g_{i(C)} g_{i(3)}^{\star} G_{(3)}^{\prime}}{G_{(3)}-G_{(C)}}
$$

Since the eigenvectors of the Christoffel matrix are unit, the third component of the second-order phase-space derivatives of the eigenvector of the Christoffel matrix can be expressed in terms of its first-order phase-space derivatives as

$$
g_{i(3)} g_{i(3)}^{\prime \star}=-g_{i(3)}^{\prime} g_{i(3)}^{\star} .
$$

We insert relations (28) and (38) into Eq. (42) and obtain

$$
\begin{aligned}
g_{i(C)} g_{i(3)}^{\prime \star}=\frac{g_{i(C)} \Gamma_{i j}^{\prime \star} g_{j(3)}}{G_{(3)}-G_{(C)}} & +\sum_{B} \frac{g_{i(C)} \Gamma_{i j}^{\prime} g_{j(B)} \Gamma_{(B 3)}^{\star}+g_{i(C)} \Gamma_{i j}^{\star} g_{j(B)} \Gamma_{(B 3)}^{\prime}}{\left(G_{(3)}-G_{(C)}\right)\left(G_{(3)}-G_{(B)}\right)} \\
& -\sum_{B} \frac{g_{i(C)} g_{i(B)} \Gamma_{(B 3)}^{\prime} \Gamma_{(33)}^{\star}+g_{i(C)} g_{i(B)} \Gamma_{(B 3)}^{\star} \Gamma_{(33)}^{\prime}}{\left(G_{(3)}-G_{(C)}\right)\left(G_{(3)}-G_{(B)}\right)},
\end{aligned}
$$




\section{Klimeš and P. Bulant}

which with definitions (25) and (26) reads

$$
g_{i(C)} g_{i(3)}^{\prime \star}=\frac{\Gamma_{(C 3)}^{\prime \star}}{G_{(3)}-G_{(C)}}+\sum_{B} \frac{\Gamma_{(C B)}^{\prime} \Gamma_{(B 3)}^{\star}+\Gamma_{(C B)}^{\star} \Gamma_{(B 3)}^{\prime}}{\left(G_{(3)}-G_{(C)}\right)\left(G_{(3)}-G_{(B)}\right)}-\frac{\Gamma_{(C 3)}^{\prime} \Gamma_{(33)}^{\star}+\Gamma_{(C 3)}^{\star} \Gamma_{(33)}^{\prime}}{\left(G_{(3)}-G_{(C)}\right)^{2}} .
$$

We insert relation (38) into identity (43) and obtain

$$
g_{i(3)} g_{i(3)}^{\prime \star}=-\sum_{C} \frac{\Gamma_{(C 3)}^{\prime} \Gamma_{(C 3)}^{\star}}{\left(G_{(3)}-G_{(C)}\right)^{2}} .
$$

Relations (45) and (46) yield expression

$$
\begin{aligned}
t_{n} g_{n(3)}^{\prime \star} & =\sum_{C} t_{(C)} \frac{\Gamma_{(C 3)}^{\prime \star}}{G_{(3)}-G_{(C)}}+\sum_{C} t_{(C)} \sum_{B} \frac{\Gamma_{(C B)}^{\prime} \Gamma_{(B 3)}^{\star}+\Gamma_{(C B)}^{\star} \Gamma_{(B 3)}^{\prime}}{\left(G_{(3)}-G_{(C)}\right)\left(G_{(3)}-G_{(B)}\right)} \\
& -\sum_{C} t_{(C)} \frac{\Gamma_{(C 3)}^{\prime} \Gamma_{(33)}^{\star}+\Gamma_{(C 3)}^{\star} \Gamma_{(33)}^{\prime}}{\left(G_{(3)}-G_{(C)}\right)^{2}}-t_{(3)} \sum_{C} \frac{\Gamma_{(C 3)}^{\prime} \Gamma_{(C 3)}^{\star}}{\left(G_{(3)}-G_{(C)}\right)^{2}}
\end{aligned}
$$

which can be inserted into relation (34).

4.6. First-order and second-order phase-space derivatives of the reference SV Hamiltonian function We insert Eq. (39) into relation (32),

$$
G_{\mathrm{SV}}^{\prime}=\frac{t_{(a)} \Gamma_{(a b)}^{\prime} t_{(b)}-\left(t_{(3)}\right)^{2} \Gamma_{(33)}^{\prime}}{t_{(S)} t_{(S)}}-2 \frac{G_{(3)}-G_{\mathrm{SV}}}{t_{(S)} t_{(S)}} t_{(3)} \sum_{N} t_{(N)} \frac{\Gamma_{(N 3)}^{\prime}}{G_{(3)}-G_{(N)}} .
$$

We insert Eqs (36), (39) and (47) into relation (34), and obtain

$$
\begin{aligned}
& G_{\mathrm{SV}}^{\prime \star}=\frac{t_{(a)} \Gamma_{(a b)}^{\prime \star} t_{(b)}-\left(t_{(3)}\right)^{2} \Gamma_{(33)}^{\prime \star}}{t_{(S)} t_{(S)}}-\frac{2\left(t_{(3)}\right)^{2}}{t_{(S)} t_{(S)}} \sum_{B} \frac{\Gamma_{(B 3)}^{\prime} \Gamma_{(B 3)}^{\star}}{G_{(3)}-G_{(B)}} \\
&+2 \frac{t_{(a)} \Gamma_{(a b)}^{\prime} t_{(b)}-\Gamma_{(33)}^{\prime} t_{(r)} t_{(r)}}{\left(t_{(S)} t_{(S)}\right)^{2}} t_{(3)} \sum_{N} t_{(N)} \frac{\Gamma_{(N 3)}^{\star}}{G_{(3)}-G_{(N)}} \\
&+2 \frac{t_{(a)} \Gamma_{(a b)}^{\star} t_{(b)}-\Gamma_{(33)}^{\star} t_{(r)} t_{(r)}}{\left(t_{(S)} t_{(S)}\right)^{2}} t_{(3)} \sum_{N} t_{(N)} \frac{\Gamma_{(N 3)}^{\prime}}{G_{(3)}-G_{(N)}} \\
&-2 \frac{G_{(3)}-G_{\mathrm{SV}}}{t_{(S)} t_{(S)}}\left(1+4 \frac{\left(t_{(3)}\right)^{2}}{t_{(S)} t_{(S)}}\right)\left(\sum_{M} t_{(M)} \frac{\Gamma_{(M 3)}^{\prime}}{G_{(3)}-G_{(M)}}\right)\left(\sum_{N} t_{(N)} \frac{\Gamma_{N 3}^{\star}}{\left.G_{(3)}-G_{(N)}\right)}\right) \\
&-2 \frac{G_{(3)}-G_{\mathrm{SV}}}{t_{(S)} t_{(S)}} t_{(3)} \sum_{C}\left(t_{(C)} \frac{\Gamma_{(C 3)}^{\prime \prime}}{G_{(3)}-G_{(C)}}+t_{(C)} \sum_{B} \frac{\Gamma_{(C B)}^{\prime} \Gamma_{(B 3)}^{\star}+\Gamma_{(C B)}^{\star} \Gamma_{(B 3)}^{\prime}}{\left(G_{(3)}-G_{(C)}\right)\left(G_{(3)}-G_{(B)}\right)}\right. \\
&\left.-t_{(C)} \frac{\Gamma_{(C 3)}^{\prime} \Gamma_{(33)}^{\star}+\Gamma_{(C 3)}^{\star} \Gamma_{(33)}^{\prime}}{\left(G_{(3)}-G_{(C)}\right)^{2}}-t_{(3)} \frac{\Gamma_{(C 3)}^{\prime} \Gamma_{(C 3)}^{\star}}{\left(G_{(3)}-G_{(C)}\right)^{2}}\right) .
\end{aligned}
$$

The colours in relation (49) will serve for referencing the individual terms of the relation in the next section. 


\section{WEIGHTING FACTORS OF THE PHASE-SPACE DERIVATIVES OF THE CHRISTOFFEL MATRIX}

\subsection{Reference SV Hamiltonian function}

We express the first-order phase-space derivatives of twice the reference SV Hamiltonian function $G_{\mathrm{SV}}$ in terms of the first-order phase-space derivatives of the Christoffel matrix,

$$
G_{\mathrm{SV}}^{\prime}=\sum_{a} \sum_{b} w_{(a b)}^{\prime} \Gamma_{(a b)}^{\prime}
$$

The weighting factors in derivative (50) obey symmetry relation

$$
w_{(a b)}^{\prime}=w_{(b a)}^{\prime} .
$$

We express the second-order phase-space derivatives of $G_{\mathrm{SV}}$ in terms of the firstorder and second-order phase-space derivatives of the Christoffel matrix,

$$
G_{\mathrm{SV}}^{\prime \star}=\sum_{a} \sum_{b} w_{(a b)}^{\prime \star} \Gamma_{(a b)}^{\prime \star}+\sum_{a} \sum_{b} \sum_{c} \sum_{d} w_{(a b c d)}^{\prime \star} \Gamma_{(a b)}^{\prime} \Gamma_{(c d)}^{\star}
$$

The weighting factors in derivative (52) obey symmetry relations

$$
w_{(a b)}^{\prime \star}=w_{(b a)}^{\prime \star}
$$

and

$$
w_{(a b c d)}^{\prime \star}=w_{(b a c d)}^{\prime \star}=w_{(a b d c)}^{\prime \star}=w_{(c d a b)}^{\prime \star} .
$$

The weighting factors in derivative (50) can be obtained from relation (48) as

$$
\begin{gathered}
w_{(A B)}^{\prime}=\frac{t_{(A)} t_{(B)}}{t_{(S)} t_{(S)}}, \\
w_{(A 3)}^{\prime}=\frac{t_{(A)} t_{(3)}}{t_{(S)} t_{(S)}}-\frac{G_{(3)}-G_{S V}}{t_{(S)} t_{(S)}} \frac{t_{(A)} t_{(3)}}{G_{(3)}-G_{(A)}},
\end{gathered}
$$

which can be expressed as

$$
w_{(A 3)}^{\prime}=\frac{t_{(A)} t_{(3)}}{t_{(S)} t_{(S)}} \frac{G_{\mathrm{SV}}-G_{(A)}}{G_{(3)}-G_{(A)}},
$$

and

$$
w_{(33)}^{\prime}=0 \text {. }
$$

Note that the weighting factors are independent of particular phase-space derivatives '. 


\section{Klimeš and P. Bulant}

The weighting factors in derivative (52) can be obtained from relation (49), and are again independent of particular phase-space derivatives ' and *. The terms with blue in relation (49) correspond to weighting factors

$$
\begin{gathered}
w_{(A B)}^{\prime \star}=w_{(A B)}^{\prime}, \\
w_{(A 3)}^{\prime \star}=w_{(A 3)}^{\prime}, \\
w_{(33)}^{\prime \star}=0 .
\end{gathered}
$$

There are no terms in relation (49) corresponding to weighting factors

$$
\begin{aligned}
& w_{(A B C D)}^{\prime \star}=0, \\
& w_{(A B 33)}^{\prime \star}=0, \\
& w_{(3333)}^{\prime \star}=0 .
\end{aligned}
$$

The second and third lines and the first term with green in relation (49) yield weighting factors

$$
w_{(A B C 3)}^{\prime \star}=\frac{t_{(A)} t_{(B)} t_{(C)} t_{(3)}}{\left(G_{(3)}-G_{(C)}\right)\left(t_{(S)} t_{(S)}\right)^{2}}-\frac{\left(G_{(3)}-G_{S V}\right)\left(t_{(A)} \delta_{B C}+t_{(B)} \delta_{A C}\right) t_{(3)}}{2\left(G_{(3)}-G_{(A)}\right)\left(G_{(3)}-G_{(B)}\right) t_{(S)} t_{(S)}} .
$$

The second and third lines and the terms with red in relation (49) yield weighting factors

$$
\begin{aligned}
w_{(A 3 C 3)}^{\prime \star} & =\frac{t_{(A)} t_{(3)} t_{(C)} t_{(3)}}{\left(t_{(S)} t_{(S)}\right)^{2}}\left(\frac{1}{G_{(3)}-G_{(A)}}+\frac{1}{G_{(3)}-G_{(C)}}\right)-\frac{\delta_{A C}\left(t_{(3)}\right)^{2}}{2 t_{(S)} t_{(S)}\left(G_{(3)}-G_{(A)}\right)} \\
& -\frac{G_{(3)}-G_{S V}}{2\left(G_{(3)}-G_{(A)}\right)\left(G_{(3)}-G_{(C)}\right)}\left[\frac{t_{(A)} t_{(C)}}{t_{(S)} t_{(S)}}\left(1+4 \frac{\left(t_{(3)}\right)^{2}}{t_{(R)} t_{(R)}}\right)-\delta_{A C} \frac{\left(t_{(3)}\right)^{2}}{t_{(S)} t_{(S)}}\right] .
\end{aligned}
$$

The second and third lines and the second term with green in relation (49) yield weighting factors

$$
w_{(A 333)}^{\prime \star}=-\frac{t_{(A)} t_{(3)}}{t_{(S)} t_{(S)}\left(G_{(3)}-G_{(A)}\right)}+\frac{G_{(3)}-G_{\mathrm{SV}}}{t_{(S)} t_{(S)}} \frac{t_{(A)} t_{(3)}}{\left(G_{(3)}-G_{(A)}\right)^{2}} .
$$

Relation (66) can be expressed as

$$
\begin{aligned}
w_{(A 3 C 3)}^{\prime \star}= & \frac{1}{G_{(3)}-G_{(A)}} \frac{1}{G_{(3)}-G_{(C)}} \\
\times & \left\{\left[\frac{G_{(3)}-G_{(A)}}{2}+\frac{G_{(3)}-G_{(C)}}{2}\right]\left[2 \frac{t_{(A)} t_{(3)} t_{(C)} t_{(3)}}{\left.t_{(S)} t_{(S)}\right)^{2}}-\frac{\delta_{A C}}{2} \frac{\left(t_{(3)}\right)^{2}}{t_{(S)} t_{(S)}}\right]\right. \\
& \left.-\left(G_{(3)}-G_{S V}\right)\left[\frac{t_{(A)} t_{(C)}}{t_{(S)} t_{(S)}}\left(\frac{1}{2}+2 \frac{\left(t_{(3)}\right)^{2}}{t_{(R)} t_{(R)}}\right)-\frac{\delta_{A C}}{2} \frac{\left(t_{(3)}\right)^{2}}{t_{(S)} t_{(S)}}\right]\right\},
\end{aligned}
$$


and finally as

$$
\begin{aligned}
& w_{(A 3 C 3)}^{\prime \star}=\frac{1}{G_{(3)}-G_{(A)}} \frac{1}{G_{(3)}-G_{(C)}} \\
& \quad \times\left[\left(G_{\mathrm{SV}}-\frac{G_{(A)}}{2}-\frac{G_{(C)}}{2}\right) \frac{\left(t_{(3)}\right)^{2}}{t_{(R)} t_{(R)}}\left(2 \frac{t_{(A)} t_{(C)}}{t_{(S)} t_{(S)}}-\frac{\delta_{A C}}{2}\right)-\frac{G_{(3)}-G_{S V}}{2} \frac{t_{(A)} t_{(C)}}{t_{(S)} t_{(S)}}\right] .
\end{aligned}
$$

Relation (67) can be expressed as

$$
w_{(A 333)}^{\prime \star}=-\frac{t_{(A)} t_{(3)}}{t_{(R)} t_{(R)}} \frac{G_{\mathrm{SV}}-G_{(A)}}{\left(G_{(3)}-G_{(A)}\right)^{2}},
$$

and finally as

$$
w_{(A 333)}^{\prime \star}=-\frac{w_{(A 3)}^{\prime}}{G_{(3)}-G_{(A)}} .
$$

\subsection{Reference SH Hamiltonian function}

We express the first-order phase-space derivatives of twice the reference $\mathrm{SH}$ Hamiltonian function $G_{\mathrm{SH}}$ in terms of the first-order phase-space derivatives of the Christoffel matrix,

$$
G_{\mathrm{SH}}^{\prime}=\sum_{a} \sum_{b} \widetilde{w}_{(a b)}^{\prime} \Gamma_{(a b)}^{\prime}
$$

The weighting factors in derivative (72) obey symmetry relation (51) analogously as the weighting factors in derivative (50).

We express the second-order phase-space derivatives of $G_{\mathrm{SH}}$ in terms of the first-order and second-order phase-space derivatives of the Christoffel matrix,

$$
G_{\mathrm{SH}}^{\prime \star}=\sum_{a} \sum_{b} \widetilde{w}_{(a b)}^{\prime \star} \Gamma_{(a b)}^{\prime \star}+\sum_{a} \sum_{b} \sum_{c} \sum_{d} \widetilde{w}_{(a b c d)}^{\prime \star} \Gamma_{(a b)}^{\prime} \Gamma_{(c d)}^{\star}
$$

The weighting factors in derivative (73) obey symmetry relations (53) and (54) analogously as the weighting factors in derivative (52).

Relations (10), (11), (28), (35) and (59)-(61) yield weighting factors

$$
\begin{gathered}
\widetilde{w}_{(11)}^{\prime \star}=\widetilde{w}_{(11)}^{\prime}=1-w_{(11)}^{\prime}, \\
\widetilde{w}_{(22)}^{\prime \star}=\widetilde{w}_{(22)}^{\prime}=1-w_{(22)}^{\prime}, \\
\widetilde{w}_{(1313)}^{\prime \star}=-\frac{1}{2\left(G_{(3)}-G_{(1)}\right)}-w_{(1313)}^{\prime \star}, \\
\widetilde{w}_{(2323)}^{\prime \star}=-\frac{1}{2\left(G_{(3)}-G_{(2)}\right)}-w_{(2323)}^{\prime \star} .
\end{gathered}
$$




\section{Klimeš and P. Bulant}

All other weighting factors in derivatives (72) and (73) are

$$
\begin{gathered}
\widetilde{w}_{(a b)}^{\prime \star}=\widetilde{w}_{(a b)}^{\prime}=-w_{(a b)}^{\prime}, \\
\widetilde{w}_{(a b c d)}^{\prime \star}=-w_{(a b c d)}^{\prime \star} .
\end{gathered}
$$

\section{CONCLUSIONS}

The SH (ordinary) and SV (extraordinary) rays in uniaxial (transversely isotropic) anisotropic media or the $\mathrm{SH}$ and $\mathrm{SV}$ reference rays in approximately uniaxial anisotropic (approximately transversely isotropic) media may represent very accurate reference rays for the coupling ray theory. To trace the $\mathrm{SH}$ and $\mathrm{SV}$ reference rays in an approximately uniaxial anisotropic medium, we need the reference $\mathrm{SH}$ and SV Hamiltonian functions. The reference SH (ordinary) and SV (extraordinary) Hamiltonian functions can be defined using the reference symmetry vector of an approximately uniaxial anisotropic medium (Kliměs, 2015, 2016a).

In this paper, we have proposed the expressions for the reference SH and SV Hamiltonian functions, and derived the expressions for their first-order and secondorder phase-space derivatives, which are necessary for ray tracing and for the equations of geodesic deviation (dynamic ray tracing). The derived equations may be applied to an anisotropic or bianisotropic medium with the symmetric stiffness or constitutive matrix, and are especially useful if the medium is close to uniaxially anisotropic (transversely isotropic).

For the sake of simplicity, we have assumed a constant reference symmetry vector of an approximately uniaxial anisotropic medium in this paper. However, the presented equations can simply be generalized to a generally heterogeneous dependence of the reference symmetry vector on the spatial coordinates using the equations by Kliměs (2015, 2016a).

Improvement of the coupling-ray-theory synthetic seismograms calculated along the $\mathrm{SH}$ and SV reference rays in an approximately uniaxial (approximately transversely isotropic) elastic medium in comparison to the anisotropic common reference rays have been demonstrated in velocity models QI2 and QI4 by Klimeš and Bulant (2014a) and Bulant and Klimers (2017), and in velocity models SC1_I and SC1_II by Klimeř and Bulant (2015) and Bulant and Kliměs (2017).

Acknowledgements: We are grateful to Einar Iversen who encouraged us in this study and then wrote a review which made it possible for us to improve the paper considerably. We also thank an anonymous reviewer for his suggestions. The research has been supported by the Grant Agency of the Czech Republic under contracts 16-01312S and 16-05237S, and by the members of the consortium "Seismic Waves in Complex 3-D Structures" (see "http:/ /sw3d.cz"). 
$S H$ and $S V$ reference rays in an approximately uniaxial generally anisotropic medium

\section{References}

Bakker P.M., 2002. Coupled anisotropic shear wave raytracing in situations where associated slowness sheets are almost tangent. Pure Appl. Geophys., 159, 1403-1417.

Bulant P. and Klimeš L., 2002. Numerical algorithm of the coupling ray theory in weakly anisotropic media. Pure Appl. Geophys., 159, 1419-1435.

Bulant P. and Klimeš L., 2008. Numerical comparison of the isotropic-common-ray and anisotropic-common-ray approximations of the coupling ray theory. Geophys. J. Int., 175, 357-374.

Bulant P. and Klimeš L., 2014. Anisotropic-ray-theory geodesic deviation and two-point ray tracing through a split intersection singularity. Seismic Waves in Complex 3-D Structures, 24, 179-187 (http://sw3d.cz).

Bulant P. and Klimeš L., 2017. Prevailing-frequency approximation of the coupling ray theory along the SH and SV reference rays in a heterogeneous generally anisotropic medium which is approximately uniaxial. Stud. Geophys. Geod., 61, 513-540.

Červený V., 1972. Seismic rays and ray intensities in inhomogeneous anisotropic media. Geophys. J. R. Astr. Soc., 29, 1-13.

Chapman C.H. and Shearer P.M., 1989. Ray tracing in azimuthally anisotropic media II. Quasi-shear wave coupling. Geophys. J., 96, 65-83.

Coates R.T. and Chapman C.H., 1990. Quasi-shear wave coupling in weakly anisotropic 3-D media. Geophys. J. Int., 103, 301-320.

Crampin S., 1981. A review of wave motion in anisotropic and cracked elastic-media. Wave Motion, 3, 343-391.

Fuki A.A., Kravtsov Yu.A. and Naida O.N., 1998. Geometrical Optics of Weakly Anisotropic Media. Gordon and Breach, Amsterdam.

Hamilton W.R., 1837. Third supplement to an essay on the theory of systems of rays. Trans. Roy. Irish Acad., 17, 1-144.

Klimeš L., 2006. Common-ray tracing and dynamic ray tracing for $\mathrm{S}$ waves in a smooth elastic anisotropic medium. Stud. Geophys. Geod., 50, 449-461.

Klimeš L., 2010. Sensitivity of electromagnetic waves to a heterogeneous bianisotropic structure. Seismic Waves in Complex 3-D Structures, 20, 207-213 (http://sw3d.cz).

Klimeš L., 2013. Relation between the propagator matrix of geodesic deviation and the second-order derivatives of the characteristic function for a general Hamiltonian function. Seismic Waves in Complex 3-D Structures, 23, 121-134 (http://sw3d.cz).

Klimeš L., 2015. Determination of the reference symmetry axis of a generally anisotropic medium which is approximately transversely isotropic. Seismic Waves in Complex 3-D Structures, 25, 177-185 (http://sw3d.cz).

Klimeš L., 2016a. Determination of the reference symmetry axis of a generally anisotropic medium which is approximately transversely isotropic. Stud. Geophys. Geod., 60, 391402.

Klimeš L., 2016b. Ray series for electromagnetic waves in static heterogeneous bianisotropic dielectric media. Seismic Waves in Complex 3-D Structures, 26, 167-182 (http:/ /sw3d.cz). 


\section{Klimeš and P. Bulant}

Klimeš L., 2016c. Ray series for electromagnetic waves in static heterogeneous bianisotropic dielectric media. In: 2016 URSI International Symposium on Electromagnetic Theory, USB Proceedings. IEEE, Washington, 331-334 (ISBN 978-1-5090-2501-5).

Klimeš L., 2017a. Rotationally invariant bianisotropic electromagnetic medium. Seismic Waves in Complex 3-D Structures, 27, 111-118 (http://sw3d.cz).

Klimeš L., 2017b. Determination of the reference symmetry axis of a generally bianisotropic electromagnetic medium which could be approximately rotationally invariant. Seismic Waves in Complex 3-D Structures, 27, 127-131 (http://sw3d.cz).

Klimeš L. and Bulant P., 2004. Errors due to the common ray approximations of the coupling ray theory. Stud. Geophys. Geod., 48, 117-142.

Klimeš L. and Bulant P., 2006. Errors due to the anisotropic-common-ray approximation of the coupling ray theory. Stud. Geophys. Geod., 50, 463-477.

Klimeš L. and Bulant P., 2012. Single-frequency approximation of the coupling ray theory. Seismic Waves in Complex 3-D Structures, 22, 143-167 (http://sw3d.cz).

Klimeš L. and Bulant P., 2014a. Prevailing-frequency approximation of the coupling ray theory for $\mathrm{S}$ waves along the $\mathrm{SH}$ and $\mathrm{SV}$ reference rays in a transversely isotropic medium. Seismic Waves in Complex 3-D Structures, 24, 165-177 (http://sw3d.cz).

Klimeš L. and Bulant P., 2014b. Anisotropic-ray-theory rays in velocity model SC1_II with a split intersection singularity. Seismic Waves in Complex 3-D Structures, 24, 189-205 (http://sw3d.cz).

Klimeš L. and Bulant P., 2015. Ray tracing and geodesic deviation of the SH and SV reference rays in a heterogeneous generally anisotropic medium which is approximately transversely isotropic. Seismic Waves in Complex 3-D Structures, 25, 187-208 (http:/ /sw3d.cz).

Klimeš L. and Bulant P., 2016. Prevailing-frequency approximation of the coupling ray theory for electromagnetic waves or elastic S waves. Stud. Geophys. Geod., 60, 419-450.

Kravtsov Yu.A., 1968. "Quasiisotropic" approximation to geometrical optics. Dokl. Acad. Nauk SSSR, 183, 74-76 (in Russian, English translation: Sov. Phys. - Doklady, 13(1969), 1125-1127).

Naida O.N., 1977. Uniform geometrical-optics approximation of linear systems along rays of variable multiplicity. Radiophys. Quantum Electron., 20, 261-271.

Naida O.N., 1979. The geometric optics of three-dimensional inhomogeneous anisotropic media. Radio Eng. Electron. Phys., 23, 8-15. 